\title{
On a new law of bone remodeling based on damage elasticity: a thermodynamic approach
}

\author{
Ahmed Idhammad ${ }^{*}$ and Abdelmounaïm Abdali
}

\author{
* Correspondence: \\ a.idhammad@gmail.com \\ Laboratory of Applied Mathematics \\ and Computer Science (LAMAI), \\ Faculty of Sciences and Technics, \\ Abdelkrim El Khattabi Avenue, \\ Marrakech, Morocco
}

\begin{abstract}
Background: Bone tissue is the main element of the human skeleton and is a dynamic tissue that is continuously renewed by bone-resorbing osteoclasts and bone-forming osteoblasts.

The bone is also capable of repairing itself and adapting its structure to changes in its load environment through the process of bone remodeling.

Therefore, this phenomenon has been gaining increasing interest in the last years and many laws have been developed in order to simulate this process.

Results: In this paper, we develop a new law of bone remodeling in the context of damaged elastic by applying the thermodynamic approach in the case of small perturbations.

The model is solved numerically by a finite difference method in the one-dimensional bone structure of a n-unit elements model.

Conclusion: In addition, several numerical simulations are presented that confirm the accuracy and effectiveness of the model.

Keywords: Numerical simulation, Thermodynamic approach, Small perturbations hypothesis, Bone remodeling, Bone density, Damage, Fatigue, Osteocyte, Elasticity, $\mathrm{N}$-unit elements
\end{abstract}

\section{Introduction}

Bone is a living material that constantly replaces old tissue with new in a process called remodeling. It is also able to respond adaptively to its environment $[1,2]$.

The bone remodeling process replaces approximately $20 \%$ of bone tissue annually; in healthy adults, bone remodeling occurs in a balanced, highly regulated manner in five phases: activation, resorption, reversal, formation, and quiescence as shown in Figure 1 [3-5].

This process is assumed to repair the microdamage and maintain bone quality; and also occurs continuously with each cycle lasting 4 to 7 months [6].

Over the past, the progress made in understanding bone remodeling, through two groups: phenomenological approach and thermodynamic approach, has been truly spectacular.

The thermodynamic approach was initiated first by chemists and was applied to continuum mechanics by Eckart and Biot around 1950. Furthermore, this approach was adopted by introducing state variables $[7,8]$ and thermodynamic potential which allows to define associated variables chosen for the study of the phenomenon [9].

(c) 2012 Idhammad and Abdali; licensee BioMed Central Ltd. This is an Open Access article distributed under the terms of the Creative Commons Attribution License (http://creativecommons.org/licenses/by/2.0), which permits unrestricted use, distribution, and reproduction in any medium, provided the original work is properly cited. 


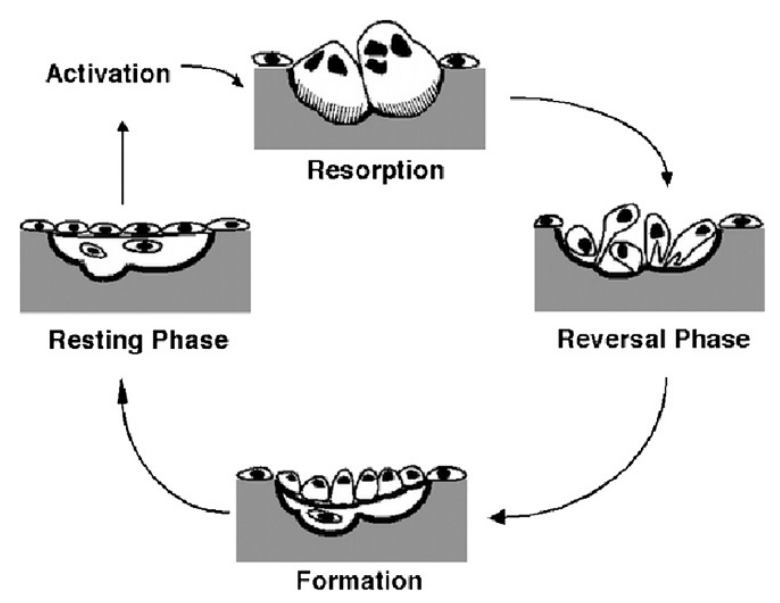

Figure 1 Bone remodeling sequence.

Many theoretical and experimental works have been published emphasizing the importance of applying this thermodynamical approach to a bone structure [10-13].

In this study, we adopt thermodynamic approach of irreversible processes to get a new law of bone remodeling taking into account the bone density described by the law of Mullender et al. [14] and the damage evolution in the bone proposed by Martin [15].

The found equation is solved by the finite difference method (FDM) in the onedimensional bone structure of a n-unit elements model.

Finally, we present some examples of numerical simulation results.

\section{Fundamental assumptions}

In order to construct a general framework for the description of the bone remodeling process, the following simplified assumptions are made:

1) The bone is considered as a linear-elastic, isotropic and inhomogeneous material.

2) The external mechanical loading acts as a stimulus for bone remodeling.

3) The n-unit elements model is applied to the damaged-bone structure in the onedimensional case.

4) The small perturbations hypothesis (displacements and their time and spatial variations are small).

5) The state coupling of damage with elastic strain.

6) The remodeling processes can be considered isothermal, adiabatic and without internal generation of heat.

7) The decoupling of the thermodynamic potential is assumed, such that:

$$
\psi=\psi\left(\varepsilon^{e}, D, \phi\right)=\psi_{e}\left(\varepsilon^{e}, D\right)+\psi_{r}(\phi)
$$

With:

$\psi_{e}\left(\varepsilon^{e}, D\right)$ is the thermodynamic potential depending on the elastic strain tensor $\varepsilon^{e}$ and the damage variable $D$.

$\psi_{r}(\phi)$ is the thermodynamic potential depending on the bone density $\phi$. 


\section{Thermodynamic approach}

The thermodynamics of irreversible processes allows the modeling of different materials behavior. This is accomplished by defining the state variables and the state potential and also the dissipation potential [16].

The general theory of adaptive damaged-elastic materials and general framework of continuum thermodynamics is considered to find a new law of bone remodeling $[10,17]$.

A general definition of the thermodynamic forces associated with the internal variables is given by:

$$
A_{k}=\rho \frac{\partial \psi}{\partial V_{k}} \quad(k=1,2, \ldots)
$$

Where conjugate forces $A_{k}$ associated with internal variables $\left(V_{\mathrm{k}}, \mathrm{k}=1,2, \ldots\right)$ by specification of the thermodynamic potential $\psi\left(\ldots, V_{k}\right)$ as shown in Table $1[17,18]$.

Within the hypothesis of small strains and small displacements, the state variables, observable and internal, are chosen in accordance with the physical mechanisms of deformation and degradation of the bone as follows [18].

Observable variables:

- $\varepsilon$ is the total strain tensor associated with the stress tensor $\sigma$.

- $T$ is the temperature associated with the specific entropy $s$.

Internal variables:

- $\varepsilon^{\mathrm{e}}$ is the elastic strain tensor associated with the stress tensor $\sigma$.

- $D$ is the damage associated with a variable $\bar{Y}$.

- $\phi$ is the bone density associated with the bone remodeling variable $R$.

Table 2 summarizes the set of variables introduced [18].

We assume a bone remodeling variable, which is characterized by:

$R>0$ in the case of the formation phase.

$R=O$ in the case of the equilibrium phase.

$R<0$ in the case of the resorption phase.

We postulate the existence of a thermodynamic potential from which the state laws can be derived $[16,18,19]$.

The state potential: $\psi=\psi\left(\varepsilon^{e}, D, \phi\right)$

Table 1 Thermodynamic variables

\begin{tabular}{lll}
\hline State variables & Internal & $\begin{array}{l}\text { Associated } \\
\text { variables }\end{array}$ \\
\hline Observable & & $s$ \\
\hline$\tau$ & & $\sigma$ \\
& $V_{k}$ & $A_{k}$ \\
\hline
\end{tabular}


Table 2 Chart of thermodynamic variables

\begin{tabular}{lll}
\hline State variables & Internal & $\begin{array}{l}\text { Associated } \\
\text { variables }\end{array}$ \\
\hline Observable & & $s$ \\
\hline$T$ & & $\sigma$ \\
$\varepsilon^{e}$ & $\sigma$ \\
$D$ & $\bar{Y}$ \\
& $\phi$ & $-R$ \\
\hline
\end{tabular}

We assume the following decoupling:

$$
\psi=\psi_{e}\left(\varepsilon^{e}, D\right)+\psi_{r}(\phi)
$$

With:

$\psi_{e}\left(\varepsilon^{e}, D\right)$ is the thermodynamic potential depending on the elastic strain tensor and the damage variable.

$\psi_{r}(\phi)$ is the thermodynamic potential depending on the bone density.

The associated variables are defined by:

$$
\begin{aligned}
& \overline{\mathrm{Y}}=\rho \frac{\partial \psi_{e}}{\partial D} \\
& -R=\rho \frac{\partial \psi_{r}}{\partial \phi}
\end{aligned}
$$

The second law of thermodynamics imposes a restriction on dissipation which can be represented in terms of the Clausius-Duhem inequality $[7,16,18]$ :

$$
\sigma: \dot{\varepsilon}-\rho \cdot \dot{\psi} \geq 0
$$

The development of the Clausius-Duhem inequality:

We note that:

$$
\rho \cdot \dot{\psi}=\rho \frac{\partial \psi_{e}}{\partial \varepsilon^{e}}: \dot{\varepsilon}^{e}+\rho \frac{\partial \psi_{e}}{\partial D} \cdot \dot{D}+\rho \frac{\partial \psi_{r}}{\partial \phi} \cdot \dot{\phi} \text { with } \varepsilon=\varepsilon^{e}
$$

and

$$
\sigma=\rho \cdot \frac{\partial \psi_{e}}{\partial \varepsilon^{e}}
$$

(the thermo-elasticity law) [17]

Inequality (3) may be written :

$$
\sigma: \dot{\varepsilon}-\rho \frac{\partial \psi_{e}}{\partial \varepsilon^{e}}: \dot{\varepsilon}^{e}-\rho \frac{\partial \psi_{e}}{\partial D} \cdot \dot{D}-\rho \frac{\partial \psi_{r}}{\partial \phi} . \dot{\phi} \geq 0
$$

Using the previous equations (1) (2) (4), we obtain:

$$
-\bar{Y} \cdot \dot{D}+R . \dot{\phi} \geq 0
$$

\section{Discussion:}

In the case of constant damage: $\dot{D}=0$ 
The inequality (5) gives + R. $\dot{\phi} \geq 0$

In the resorption area:

$\dot{\phi} \leq 0$ and $\mathrm{R}<0$

$$
=>+R \cdot \dot{\phi} \geq 0
$$

In the formation area:

$\dot{\phi} \geq 0$ and $\mathrm{R}>0$

$$
=>+R \cdot \dot{\phi} \geq 0
$$

Equilibrium area (dead zone):

$$
\begin{aligned}
\dot{\phi} & =0 \text { and }+ \text { R. } \dot{\phi}=0 \\
& =>+ \text { R. } \dot{\phi} \geq 0
\end{aligned}
$$

We can conclude that the inequality (5) is verified in accordance with the law of bone remodeling as shown in Figure 2 [20].

The conservation of energy equation:

The first principle of thermodynamics gives:

$$
\rho . \dot{e}=\sigma: \dot{\varepsilon}^{e}+r-\operatorname{div} \vec{q}
$$

$[17,21]$

With:

- $\mathrm{e}$ is the specific internal energy.

- $r$ is the internal heat source.

- $\mathrm{q}$ is the heat flux.

We replaced $\rho \dot{e}$ by the expression derived from $e=\psi+$ T.s $[17,21]$

$$
\rho \cdot \dot{e}=\rho \cdot \dot{\psi}+\rho . \dot{T} \cdot s+\rho . T \cdot \dot{s}
$$

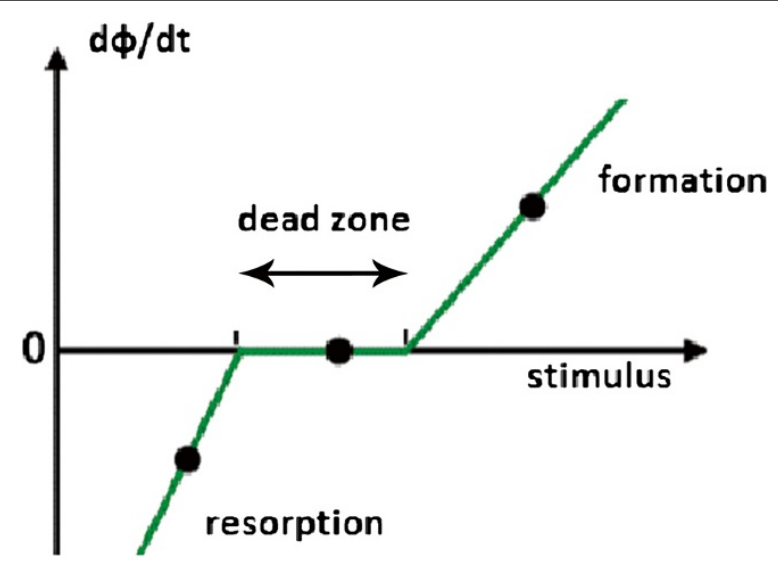

Figure 2 Law of bone remodeling. 
and $\rho \dot{\psi}$ by its expression as a function of the state variables and the associated variables.

$$
\rho \cdot \dot{\psi}=\sigma: \dot{\varepsilon}^{e}+\bar{Y} \cdot \dot{D}-R \cdot \dot{\phi}
$$

We introduce the specific heat (capacity) defined by:

$\mathrm{C}=\mathrm{T} \frac{\partial \mathrm{s}}{\partial \mathrm{T}}[16,17]$ and taking into account Fourier's law $\vec{q}=-k \cdot \operatorname{grad} T[17]$

We obtain:

$$
\operatorname{div} \vec{q}=-k \cdot \operatorname{div}(\operatorname{grad} T)=-k \cdot \Delta T
$$

Using the previous equations (6) (7) (8) (9), we can get:

$$
\overline{\mathrm{Y}} \cdot \dot{D}-R . \dot{\phi}+\rho \dot{T} . s+\rho . T . \dot{s}=r+k . \Delta T
$$

\section{Assumptions:}

The classical heat equation corresponds to a process: [17]

- Without internal generation of heat created by the external sources: $r=0$.

- With adiabatic evolution: $k \cdot \Delta T=0$.

- With isothermal transformation: $\dot{T}=0$ Therefore $s=\frac{C}{T} \cdot \dot{T}=0$ Then $\dot{s}=0$.

Equation (10) may be written:

$$
\overline{\mathrm{Y}} \cdot \dot{D}-R \cdot \dot{\phi}=0
$$

Then,

$$
R=\bar{Y} \cdot \frac{\dot{D}}{\dot{\phi}}
$$

We have: $\bar{Y}=-Y$

Where

$$
Y=\frac{1}{2} \cdot E \cdot \dot{\varepsilon}^{e}: \dot{\varepsilon}^{e}
$$

(the strain energy release rate) [17]

We have also: $\rho \cdot \psi_{e}=\frac{1}{2} \cdot(1-D) \cdot E \cdot \dot{\varepsilon}^{e}: \dot{\varepsilon}^{e}$ (the strain energy) [17] and the equivalent constraint $\sigma_{e q}$ is written by $\sigma_{e q}=\rho \frac{\partial \psi_{e}}{\partial \varepsilon^{e}}=(1-D) \cdot E \cdot \dot{\varepsilon}^{e}$ (The thermo-elasticity law)

Then,

$$
\dot{\varepsilon}=\frac{\sigma_{e q}}{(1-D) \cdot E}
$$

With: $\sigma_{e q}=\sigma$ in the one-dimensional case.

Using the previous equations (12) (13), equation (11) may be written:

$$
R=-\frac{\sigma^{2}}{2 E \cdot(1-D)^{2}} \cdot \frac{\dot{D}}{\dot{\phi}}
$$


The Young's modulus of the bone which is an isotropic material and inhomogeneous is expressed as:

$E=(1-D) \cdot E_{0}$ with $E_{0}=c \cdot \phi^{\alpha}[22,23]$

$\mathrm{c}=100$ and $\alpha=3$ are two constants characteristic of the bone

Then,

$$
E=(1-D) \cdot c . \phi^{\alpha}
$$

Equation (14) may be written:

$$
R(\phi, D)=-\frac{\sigma^{2}}{2 c} \cdot \frac{\dot{D}}{(1-D)^{3}} \cdot \frac{1}{\phi^{\alpha} \dot{\phi}}
$$

This equation represents the new law of bone remodeling developed by applying the thermodynamic approach in the context of damaged elastic.

In this study, we introduce the law of damage developed by Martin [15] which shows that the damage in human cortical bone can grow exponentially until the fatigue failure $[15,24,25]$.

The evolution law for the damage is expressed as:

$D=D_{0} \cdot e^{f_{d} t}$ Then, $\frac{\partial D}{\partial t}=\dot{D}=f_{d} D$

With:

$f_{d}$ : the fatigue life of the bone devoid of the remodeling [26]

\section{$D_{0}:$ the initial damage}

$t$ : the time

Finally, the new law of bone remodeling may be written as:

$$
R(\phi, D)=-\frac{\sigma^{2} f_{d}}{2 c} \cdot \frac{D}{(1-D)^{3}} \cdot \frac{1}{\phi^{\alpha} \dot{\phi}}
$$

\section{Numerical resolution}

The new law of bone remodeling (Equation 15) was solved numerically by dividing it into three parts:

1. a constant $\frac{\sigma^{2} f_{d}}{2 c}$

2. a damage function $\frac{D}{(1-D)^{3}}$

3. a function of bone density $\frac{1}{\phi^{\alpha} \phi}$

In this study, we use the law of bone density proposed by Mullender et al. [14]:

$$
\frac{\partial \phi_{i}}{\partial t}=\tau \sum_{k=1}^{k=m} e^{-\frac{d\left(i, I_{k}\right)}{d}}\left(\frac{S^{k}}{\phi_{k}^{\beta}}-S_{r e f}\right)
$$

With :

$-\phi_{\min } \leq \phi \leq \phi_{\max }$

- $\phi_{\min }$ is the density of completely resorbed bone 


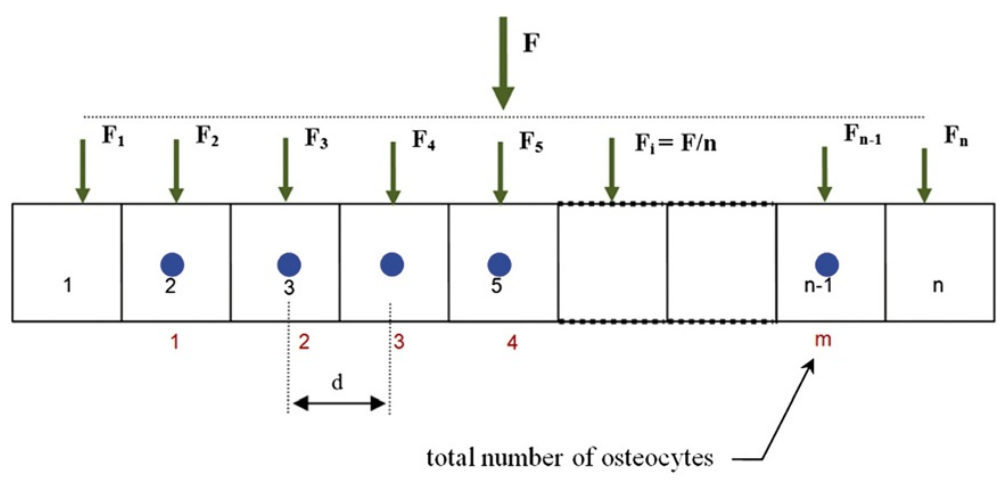

Figure 3 Model geometry with $\mathbf{n}$-unit elements.

- $\phi_{\max }$ is the maximum density defined for a compact bone

- $\tau$ is a positive constant related to the reaction time of bone tissue (constant of bone remodeling)

$-1 \leq \mathrm{i} \leq \mathrm{n}$

- $\phi_{i}$ density of bone tissue of element $i$

- $m(m \leq n)$ is the total number of osteocytes in the solid

- $I_{k}(1 \leq k \leq m)$ corresponds to the series of numbers of the elements containing an

osteocyte

- $S_{k}$ represents the density of deformation energy in $I_{k}$

- $S_{\text {ref }}$ reference stimulus value

- $\beta$ is a parameter reflecting the intensity of the stimulus cell

- $d$ is the normalization factor limiting the area of influence of osteocyte

- $d\left(i, I_{k}\right)$ is the distance between the centers of geometric element $i$ and the element $I_{k}$

We discretize into $\mathrm{n}$-unit elements a bone fragment and we apply a compressive force evenly distributed over the various units (Figure 3) [23,27].

To solve the previous equation (16), we use the method of finite difference with an implicit scheme and the fixed point method $[23,27,28]$.

Table 3 Values of the parameters used during the numerical simulations

\begin{tabular}{llll}
\hline Data & Symbol & Values & Units \\
\hline Maximum density & $\phi_{\max }$ & 1.75 & $\mathrm{~g} / \mathrm{cm}^{3}$ \\
Minimal density & $\phi_{\min }$ & 0.01 & $\mathrm{~g} / \mathrm{cm}^{3}$ \\
Initial density & $\phi_{0}$ & 0.6 & $\mathrm{~g} / \mathrm{cm}^{3}$ \\
The step of time & $\Delta \mathrm{t}$ & $5.10^{-3}$ & $\mathrm{UT}$ \\
The total force & $\mathrm{F}$ & 10 & $\mathrm{~N}$ \\
The distance between 2 centers & $\mathrm{d}$ & 25 & $\mathrm{~mm}$ \\
Reference stimulus value & $\mathrm{S}_{\mathrm{ref}}$ & 0.04 & $\mathrm{MPA}$ \\
The fatigue life of the bone & $\mathrm{f}_{\mathrm{d}}$ & 3 & years \\
$\mathrm{n}$-unit elements of the bone fragment & & 50 & \\
\hline
\end{tabular}

$m(m<n)$ is the total number of osteocytes in the bone fragment. Constants $a=3 \beta=0.5 D_{0}=0.8 c=100 \tau=1$. 
We obtain:

$$
\begin{aligned}
& \frac{\phi_{i}^{n+1}-\phi_{i}^{n}}{\Delta t}=\tau \sum_{k=1}^{k=m} e^{-\frac{d\left(i, I_{k}\right)}{d}}\left(\frac{S^{k}}{\phi_{k}^{\beta, n+1}}-S_{r e f}\right) \quad i=1,2, n \\
& \phi^{0}=\phi_{0}
\end{aligned}
$$

\section{Results and discussion}

We simulated the case of a uniform distribution of the osteocyte cells, and of another heterogeneous case. The values of the parameters used during the numerical simulations are given in Table $3[23,28]$.

Numerical results are shown in Figure 4.

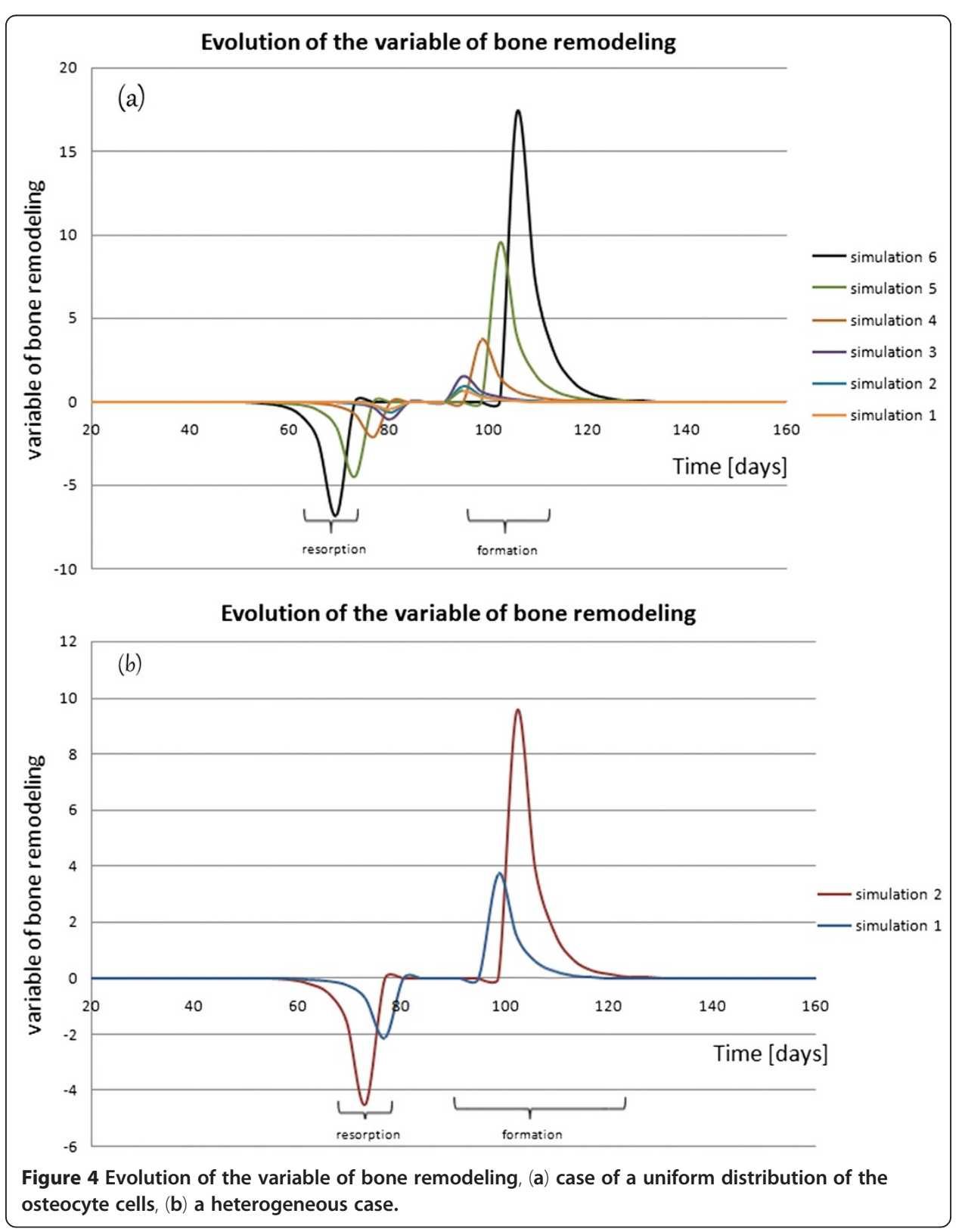


Figure 4 shows the temporal evolution of the variable of bone remodeling in the case of a uniform distribution of the osteocyte cells $(n=m=50)$, and of another heterogeneous case ( $\mathrm{n} \neq \mathrm{m}$ with $\mathrm{m}=30$ in the central package of the osteocytes).

The curves consists of three key periods. The first period of the curves corresponds to the resorption phase, where the variable of the bone remodeling was negative. The second period exhibits the formation phase, where the variable of the bone remodeling was positive. The third period defined as the interval between the resorption phase and the formation phase, which the variable of the bone remodeling reached zero.

The resorption phase takes approximately 18 days, which is then followed by an equilibrium phase that can last for up to 10 days and finally by the formation phase from 17 to 35 days. This is in agreement with results from the literature $[6,29,30]$.

By comparing the curves in Figure 4 to the graph proposed by Terrier et al. [20], we see a good agreement. Furthermore, the curves that are found have a nonlinear shape.

\section{Conclusion}

In this paper, we proposed a thermodynamic approach in small perturbations for bone remodeling process.

The adopted model takes into consideration both the bone density and the damage and gives a new law of bone remodeling. Then, the governing equation of the process was solved by the finite difference method in the one-dimensional bone structure with n-unit elements model.

The numerical results obtained are in accordance with the experimental results found in the literature.

Competing interest

The authors declare that they have no competing interests.

Authors' contributions

Both authors contributed to writing and improving the paper and approved the final manuscript.

Authors' information

Ahmed Idhammad - He received his engineering degree status at the National School of Mineral Industry in Rabat, Morocco. He is curzently a Doctoral student at the Faculty of Sciences and Technics in Marrakech, Morocco. His research interests are numerical simulation, biomechanics, bone remodeling, thermodynamic, fatigue and damage. Abdelmounaïm Abdali - PhD in Solid Mechanics and Structures in University of Amiens in 1996, France. He is a Professor in computer science at the University Cadi Ayyad, Faculty of Sciences and Technics, Marrakech, Morocco. Member at the Laboratory of Applied Mathematics and Computer Science (LAMAI) Marrakech, Morocco. His research interests are numerical simulation, biomechanics, bone remodeling and damage, computer science, DTN Network.

\section{Acknowledgements}

We are particularly grateful to the editor in chief Dr. Paul S. Agutter for his thoughtful suggestions for improving the language and style of the manuscript.

We equally thankful to all members of our laboratory team for stimulating discussions.

Received: 26 September 2012 Accepted: 18 November 2012

Published: 29 November 2012

\section{References}

1. Rüberg T: Computer Simulation of Adaptive Bone Remodeling. Spain: Master's thesis, Technische Universität Braunschweig, Centro Politécnico Superior, Universidad de Zaragoza; 2003.

2. García-Aznar JM, Rueberg T, Doblaré M: A bone remodeling model coupling microdamage growth and repair by 3D BMU-activity. Biomech Model Mechanobiol 2005, 4:147-167.

3. Comfort P, Abrahamson E: Sports Rehabilitation and Injury Prevention. 1st edition. UK: Wiley-Blackwell Publishers, John Wiley \& Sons Ltd; 2010:114-115.

4. Mohri $\mathrm{T}$, Hanada $\mathrm{K}$, Ozawa $\mathrm{H}$ : Coupling of resorption and formation on bone remodeling sequence in orthodontic tooth movement: A histochemical study. J Bone Miner Metab 1991, 9:57-69.

5. Raggatt L, Partridge NC: Cellular and Molecular Mechanisms of Bone Remodeling. J Biol Chem 2010, 285:25103-25108. 
6. Riggs BL, Parfitt AM: Drugs Used to Treat Osteoporosis: The Critical Need for a Uniform Nomenclature Based on Their Action on Bone Remodeling. J Bone Miner Res 2005, 20:177-184.

7. Coleman BD, Gurtin ME: Thermodynamics with internal state variables. J Chem Phys 1967, 47:597-613.

8. Coleman BD, Noll W: The thermodynamic of elastic materials with heat conduction and viscosity. Arch Ration Mech Anal 1963, 13:167-178.

9. Lemaitre J, Chaboche JL: Mechanics of solid materials. UK: Cambridge University Press; 1990.

10. Ramtani S, Zidi M: A theoretical model of the effect of continuum damage on a bone adaptation model. J Biomech 2001, 34:471-479.

11. Doblaré M, Garcıa JM, Gomez MJ: Modelling bone tissue fracture and healing: a review. Eng Fract Mech 2004, 71:1809-1840.

12. Kuhl E, Steinmann P: Theory and numerics of geometrically non-linear open system mechanics. Int I Numer Meth Eng 2003, 58:1593-1615.

13. Hoger A, Lubarda VA: On the mechanics of solids with a growing mass. Int I Solids Struct 2002, 39:4627-4664.

14. Mullender $M$, Huiskes $R$, Weinans $H$ : A physiological approach to the simulation of bone remodeling as self organizational control process. J Biomech 1994, 27:1389-1394.

15. Martin RB: Fatigue damage, remodeling, and the minimization of skeletal weight. J Theor Biol 2003, 220:271-276.

16. Lemaitre J, Desmorat R: Engineering Damage Mechanics. Berlin: Springer; 2005.

17. Lemaitre J, Chaboche JL, Benallal A, Desmorat R: Mécanique des matériaux solides. Paris: Dunod; 2009.

18. Lemaitre J: A Course on Damage Mechanics. NY: Springer-Verlag; 1992.

19. Barbero EJ, Greco F, Lonetti P: Continuum Damage-healing Mechanics with Application to Self-healing Composites. Int J Damage Mech 2005, 14:51-81.

20. Terrier A, Rakotomanana RL, Ramaniraka AN, Leyvraz PF: Adaptation models of anisotropic bone. Comput Meth Biomech Biomed Eng 1997, 1:47-59.

21. Wriggers P: Computational Contact Mechanics. 2nd edition. NY: Springer-Verlag; 2006.

22. Currey JD: The effect of porosity and mineral content on theYoung's modulus elasticity of compact bone. J Biomech 1988, 21:131-139.

23. Idhammad A, Abdali A, Bussy P: Numerical simulation of the process of bone remodeling in the context of damaged elastic. Int J Adv Sci Tech 2011, 37:87-98.

24. Carter DR, Hayes WC, Schurman DJ: Fatigue life of compact bone-II. Effects of microstructure and density. J Biomech 1976, 9:211-214

25. Pattin CA, Caler WE, Carter DR: Cyclic mechanical property degradation during fatigue loading on cortical bone. J Biomech 1996, 29:69-79.

26. Taylor D, Kuiper JH: The prediction of stress fractures using a stressed volume concept. J Orthop Res 2001, 19:919-926.

27. Abdali A, Almoatassime H, Errafay A: Extension of the law of Mullender within the viscoelastic framework for the digital simulation of the process of bone remodeling. Int J Math Stat 2010, 6:1-10.

28. Idhammad A, Abdali A: Numerical simulation by finite difference of the problem of bone remodeling: case of elasticity with damage. In Proceedings of the International Congress on Numerical Analysis and Scientific Computing with Applications in Sciences and Engineering: 19-20 April 2011. Settat, Morocco:; 2011:183-186.

29. Lee TC, Staines A, Taylor D: Bone adaptation to load: microdamage as a stimulus for bone remodelling. J Anat 2002, 201:437-446

30. Komarova SV, Smith RJ, Dixon SJ, Sims SM, Wahl LM: Mathematical model predicts a critical role for osteoclast autocrine regulation in the control of bone remodeling. Bone 2003, 33:206-215.

doi:10.1186/1742-4682-9-51

Cite this article as: Idhammad and Abdali: On a new law of bone remodeling based on damage elasticity: a thermodynamic approach. Theoretical Biology and Medical Modelling 2012 9:51.

\section{Submit your next manuscript to BioMed Central and take full advantage of:}

- Convenient online submission

- Thorough peer review

- No space constraints or color figure charges

- Immediate publication on acceptance

- Inclusion in PubMed, CAS, Scopus and Google Scholar

- Research which is freely available for redistribution

Submit your manuscript at www.biomedcentral.com/submit 